\title{
ON THE WELL-POSEDNESS AND REGULARITY OF THE WAVE EQUATION WITH VARIABLE COEFFICIENTS*
}

\author{
BaO-Zhu GuO ${ }^{1,2}$ AND Zhi-Xiong Zhang ${ }^{1,3}$
}

\begin{abstract}
An open-loop system of a multidimensional wave equation with variable coefficients, partial boundary Dirichlet control and collocated observation is considered. It is shown that the system is well-posed in the sense of D. Salamon and regular in the sense of G. Weiss. The Riemannian geometry method is used in the proof of regularity and the feedthrough operator is explicitly computed.
\end{abstract}

Mathematics Subject Classification. 35J50, 93C20, 93C25.

Received January 12, 2006. Revised May 25, 2006.

Published online September 5, 2007.

\section{INTRODUCTION AND MAIN RESULTS}

In the last two decades, the class of well-posed and regular linear systems has been studied extensively [5,23]. It has been demonstrated that this class of systems is quite general: it covers many control systems described by partial differential equations with actuators and sensors supported at isolated points, sub-domains, or on a part of the boundary of the spatial region. More importantly, this class of infinite-dimensional systems, although the input and output operators are allowed to be unbounded, may possess many properties that are parallel in many ways to those of finite-dimensional systems.

In particular, the concept of "regularity" is a completely new concept in this framework. However, while the abstract theory has been quite fruitful, these properties are rarely checked for control systems described by partial differential equations [4]. The well-posedness and regularity of a multidimensional heat equation with both Dirichlet and Neumann type boundary controls were established in [3]. For a wave equation with constant coefficients, boundary Dirichlet input and collocated output on a bounded open domain of $\mathbb{R}^{n}$ with smooth boundary, the well-posedness was proved in [1] and the regularity was proved recently in [11]. The regularity of multidimensional Schrödinger and Euler-Bernoulli equations with certain types of control and observation were shown in [9] and [10], respectively. Other results on the well-posedness and regularity of control systems described by multidimensional partial differential equations can be found in [20,22], etc.

Most of the aforementioned multidimensional partial differential equations are with constant coefficients. In this paper, we generalize the results of $[1,11]$ to the variable coefficients case. The system is described by the

\footnotetext{
Keywords and phrases. Wave equation, transfer function, well-posed and regular system, boundary control and observation.

* Supported by the National Natural Science Foundation of China and the National Research Foundation of South Africa.

1 Academy of Mathematics and Systems Science, Academia Sinica, Beijing 100080, P.R. China; bzguo@iss.ac.cn

2 School of Computational and Applied Mathematics, University of the Witwatersrand, Wits 2050, Johannesburg, South Africa.

3 Graduate University of Chinese Academy of Sciences, Beijing 100049, P.R. China.
} 
following wave equation with variable coefficients, partial boundary Dirichlet control and collocated observation:

$$
\left\{\begin{array}{l}
w_{t t}(x, t)-\sum_{i, j=1}^{n} \frac{\partial}{\partial x_{i}}\left(a_{i j}(x) \frac{\partial w(x, t)}{\partial x_{j}}\right)=0, x=\left(x_{1}, x_{2}, \ldots, x_{n}\right) \in \Omega, t>0 \\
w(x, t)=0, x \in \Gamma_{1}, t>0 \\
w(x, t)=u(x, t), x \in \Gamma_{0}, t>0 \\
y(x, t)=-\frac{\partial \mathcal{A}^{-1} w_{t}(x, t)}{\partial \nu_{\mathcal{A}}}, x \in \Gamma_{0}, t>0
\end{array}\right.
$$

where $\Omega \subset \mathbb{R}^{n}(n \geq 2)$ is an open bounded region with smooth boundary $\partial \Omega=: \Gamma=\overline{\Gamma_{0}} \cup \overline{\Gamma_{1}}$. $\Gamma_{0}, \Gamma_{1}$ are disjoint parts of the boundary relatively open in $\partial \Omega$ with $\operatorname{int}\left(\Gamma_{0}\right) \neq \emptyset$.

$$
\mathcal{A} w(x, t):=-\sum_{i, j=1}^{n} \frac{\partial}{\partial x_{i}}\left(a_{i j}(x) \frac{\partial w(x, t)}{\partial x_{j}}\right), D(\mathcal{A})=H^{2}(\Omega) \cap H_{0}^{1}(\Omega)
$$

and for some constant $a>0$,

$$
\begin{aligned}
a_{i j}(x) & =a_{j i}(x) \in C^{\infty}\left(\mathbb{R}^{n}\right), \sum_{i, j=1}^{n} a_{i j}(x) \xi_{i} \overline{\xi_{j}} \geq a|\xi|^{2}, \forall x \in \Omega, \xi=\left(\xi_{1}, \xi_{2}, \ldots, \xi_{n}\right) \in \mathbb{C}^{n}, \\
\nu_{\mathcal{A}} & :=\left(\sum_{k=1}^{n} \nu_{k} a_{k 1}(x), \sum_{k=1}^{n} \nu_{k} a_{k 2}(x), \ldots, \sum_{k=1}^{n} \nu_{k} a_{k n}(x)\right), \frac{\partial}{\partial \nu_{\mathcal{A}}}:=\sum_{i, j=1}^{n} \nu_{i} a_{i j}(x) \frac{\partial}{\partial x_{j}},
\end{aligned}
$$

where $\nu=\left(\nu_{1}, \nu_{2}, \ldots, \nu_{n}\right)$ is the unit normal of $\partial \Omega$ pointing towards the exterior of $\Omega$. $u$ is the input function (or control) and $y$ is the output function (or observation).

Let $\mathcal{H}=L^{2}(\Omega) \times H^{-1}(\Omega)$ and $U=L^{2}\left(\Gamma_{0}\right)$. The following theorem is the generalization of Proposition 2.2 of [1] for the system (1.1) with constant coefficients.

Theorem 1.1. Let $T>0,\left(w_{0}, w_{1}\right) \in \mathcal{H}$ and $u \in L^{2}(0, T ; U)$. Then there exists a unique solution $\left(w, w_{t}\right) \in$ $C([0, T] ; \mathcal{H})$ to the system $(1.1)$, which satisfies $w(\cdot, 0)=w_{0}$ and $w_{t}(\cdot, 0)=w_{1}$. Moreover, there exists a constant $C_{T}>0$, independent of $\left(w_{0}, w_{1}, u\right)$, such that

$$
\left\|\left(w(\cdot, T), w_{t}(\cdot, T)\right)\right\|_{\mathcal{H}}^{2}+\|y\|_{L^{2}(0, T ; U)}^{2} \leq C_{T}\left[\left\|\left(w_{0}, w_{1}\right)\right\|_{\mathcal{H}}^{2}+\|u\|_{L^{2}(0, T ; U)}^{2}\right] .
$$

Theorem 1.1 implies that the open-loop system (1.1) is well-posed in the sense of D. Salamon with the state space $\mathcal{H}$ and with the input and output space $U$ [11]. From this result and Theorem 2.2 of [2] (see also Th. 3 of [8]), we know that the system (1.1) is exactly controllable in some time interval $[0, T]$ if and only if its closed-loop system under the output proportional feedback $u=-k y, k>0$ is exponentially stable. We can thereby say that the conditions for the exponential stabilization [15] and for the exact controllability [19] of the wave equation with constant coefficients should be the same. Moreover, for the case of variable coefficients, the condition for the exact controllability stated in Theorem 1.1 of [24] is also the condition for the exponential stability of the closed-loop system (1.1) under the feedback $u=-k y, k>0$. This fact leads to the following interesting example that comes from [24] and was studied numerically in [7].

Example 1.1. Let $n=2$ and

$$
a_{11}\left(x_{1}, x_{2}\right)=a_{22}\left(x_{1}, x_{2}\right)=\left(1+x_{1}^{2}+x_{2}^{2}\right)^{2}, a_{12}\left(x_{1}, x_{2}\right)=a_{21}\left(x_{1}, x_{2}\right)=0 .
$$


Suppose $\partial B_{1} \subset \Omega$, where $B_{1}$ is the unit ball of $\mathbb{R}^{2}$. From Example 4.1 of [24] we know that in this case, the system (1.1) is not exactly controllable even if the control is imposed on the whole boundary: $\Gamma_{0}=\partial \Omega$. By the equivalence between exact controllability and exponential stability just explained above, we can now say that for this example, there exists an initial datum $\left(w(\cdot, 0), w_{t}(\cdot, 0)\right) \in \mathcal{H}$ for which the closed-loop system (1.1) under the output proportional feedback $u=-k y$ is not exponentially stable for any $k>0$.

Theorem 1.2. The system (1.1) is regular in the sense of Weiss [21]. More precisely, if $w(\cdot, 0)=w_{t}(\cdot, 0)=0$ and $u(\cdot, t) \equiv u(\cdot) \in U$ is a step input, then the corresponding output $y$ satisfies

$$
\left.\left.\lim _{\sigma \rightarrow 0} \int_{\Gamma_{0}}\left|\frac{1}{\sigma} \int_{0}^{\sigma} y(x, t) \mathrm{d} t-\right| \nu_{\mathcal{A}}(x)\right|_{g} u(x)\right|^{2} \mathrm{~d} x=0,
$$

where

$$
\left|\nu_{\mathcal{A}}(x)\right|_{g}^{2}=\sum_{i, j=1}^{n}\left(g_{i j}(x) \sum_{k=1}^{n} \nu_{k} a_{k i}(x) \sum_{l=1}^{n} \nu_{l} a_{l j}(x)\right),\left(g_{i j}(x)\right)=A(x)^{-1}, A(x)=\left(a_{i j}(x)\right), \forall x \in \Gamma .
$$

Theorems 1.1 and 1.2 ensure that the system (1.1) is a well-posed regular linear system with feedthrough operator $(D u)(x)=\left|\nu_{\mathcal{A}}(x)\right|_{g} u(x)$ for any $u \in U$. The main contributions of this paper are: (a) generalizing the results of $[1,11]$ for the system $(1.1)$ where the coefficients are constant to the variable ones; (b) much simplifying the regularity proof of [11] by using multiplier method on Riemannian manifolds.

The remaining part of the paper are organized as follows. In Section 2, we cast the system (1.1) into an abstract setting studied in [2] and [8]. The proofs of Theorems 1.1 and 1.2 will be presented in Sections 3 and 4, respectively.

\section{Collocated formulation of the system (1.1)}

Let $H=H^{-1}(\Omega)$ be the dual space of the Sobolev space $H_{0}^{1}(\Omega)$ with usual inner product. Let $A$ be the positive self-adjoint operator in $H$ induced by the bilinear form $a(\cdot, \cdot)$ defined by

$$
\langle A f, g\rangle_{H^{-1}(\Omega) \times H_{0}^{1}(\Omega)}=a(f, g)=\sum_{i, j=1}^{n} \int_{\Omega} a_{i j}(x) \frac{\partial f}{\partial x_{j}} \frac{\overline{\partial g}}{\partial x_{i}} \mathrm{~d} x, \forall f, g \in H_{0}^{1}(\Omega)
$$

where $a_{i j}(x)$ are given by $(1.2)$.

By means of the Lax-Milgram theorem, $A$ is a canonical isomorphism from $D(A)=H_{0}^{1}(\Omega)$ onto $H$. It is easy to show that $A f=\mathcal{A} f$ whenever $f \in H^{2}(\Omega) \cap H_{0}^{1}(\Omega)$ and that $A^{-1} g=\mathcal{A}^{-1} g$ for any $g \in L^{2}(\Omega)$. Hence $A$ is an extension of $\mathcal{A}$ to the space $H_{0}^{1}(\Omega)$.

It can be easily shown that $D\left(A^{1 / 2}\right)=L^{2}(\Omega)$ and $A^{1 / 2}$ is an isomorphism from $L^{2}(\Omega)$ onto $H$. Define the map $\Upsilon \in \mathcal{L}\left(L^{2}\left(\Gamma_{0}\right), H^{1 / 2}(\Omega)\right)[15]$ by $\Upsilon u=v$ if and only if

$$
\left\{\begin{array}{l}
\mathcal{A} v(x)=0, x \in \Omega \\
v(x)=0, x \in \Gamma_{1} ; v(x)=u(x), x \in \Gamma_{0}
\end{array}\right.
$$

By virtue of the above map, one can write (1.1) as

$$
\ddot{w}+A(w-\Upsilon u)=0 .
$$

Since $D(A)$ is dense in $H$, so is $D\left(A^{1 / 2}\right)$. We identify $H$ with its dual $H^{\prime}$. Then the following relations hold:

$$
D\left(A^{1 / 2}\right) \hookrightarrow H=H^{\prime} \hookrightarrow\left(D\left(A^{1 / 2}\right)\right)^{\prime} .
$$


An extension $\tilde{A} \in \mathcal{L}\left(D\left(A^{1 / 2}\right),\left(D\left(A^{1 / 2}\right)\right)^{\prime}\right)$ of $A$ is defined by

$$
\langle\tilde{A} f, g\rangle_{\left(D\left(A^{1 / 2}\right)\right)^{\prime} \times D\left(A^{1 / 2}\right)}=\left\langle A^{1 / 2} f, A^{1 / 2} g\right\rangle_{H}, \forall f, g \in D\left(A^{1 / 2}\right) .
$$

So $(2.2)$ can be further written in $\left(D\left(A^{1 / 2}\right)\right)^{\prime}$ as

$$
\ddot{w}+\tilde{A} w+B u=0
$$

where $B \in \mathcal{L}\left(U,\left(D\left(A^{1 / 2}\right)\right)^{\prime}\right)$ is given by

$$
B u=-\tilde{A} \Upsilon u, \forall u \in U
$$

Define $B^{*} \in \mathcal{L}\left(D\left(A^{1 / 2}\right), U\right)$ by

$$
\left\langle B^{*} f, u\right\rangle_{U}=\langle f, B u\rangle_{D\left(A^{1 / 2}\right) \times\left(D\left(A^{1 / 2}\right)\right)^{\prime}}, \forall f \in D\left(A^{1 / 2}\right), u \in U .
$$

Then for any $f \in D\left(A^{1 / 2}\right)$ and $u \in C_{0}^{\infty}\left(\Gamma_{0}\right)$, we have

$$
\begin{aligned}
\langle f, B u\rangle_{D\left(A^{1 / 2}\right) \times\left(D\left(A^{1 / 2}\right)\right)^{\prime}} & =\left\langle f, \tilde{A} \tilde{A}^{-1} B u\right\rangle_{D\left(A^{1 / 2}\right) \times\left(D\left(A^{1 / 2}\right)\right)^{\prime}}=\left\langle A^{1 / 2} f, A^{1 / 2} \tilde{A}^{-1} B u\right\rangle_{H} \\
& =-\left\langle A^{1 / 2} f, A^{1 / 2} \Upsilon u\right\rangle_{H}=-\langle f, \Upsilon u\rangle_{L^{2}(\Omega)} \\
& =-\left\langle\mathcal{A} \mathcal{A}^{-1} f, \Upsilon u\right\rangle_{L^{2}(\Omega)}=\left\langle\frac{\partial \mathcal{A}^{-1} f}{\partial \nu_{\mathcal{A}}}, u\right\rangle_{U} .
\end{aligned}
$$

In the last step, we have used the fact

$$
\sum_{i, j=1}^{n} \int_{\Omega} a_{i j} \frac{\partial v}{\partial x_{j}} \frac{\overline{\partial \phi}}{\partial x_{i}} \mathrm{~d} x=0, \forall \phi \in H_{0}^{1}(\Omega)
$$

which holds for any classical solution $v$ of $(2.1)$. Since $C_{0}^{\infty}\left(\Gamma_{0}\right)$ is dense in $U=L^{2}\left(\Gamma_{0}\right)$, we finally obtain that

$$
B^{*}=\left.\frac{\partial \mathcal{A}^{-1}}{\partial \nu_{\mathcal{A}}}\right|_{\Gamma_{0}}
$$

Now, we have formulated the open-loop system (1.1) into an abstract form of a second-order system in the state space $\mathcal{H}=L^{2}(\Omega) \times H^{-1}(\Omega)$ :

$$
\left\{\begin{array}{l}
\ddot{w}(t)+\tilde{A} w(t)+B u(t)=0 \\
y(t)=-B^{*} \dot{w}(t)
\end{array}\right.
$$

where $B$ and $B^{*}$ are defined by (2.4) and (2.6), respectively. The abstract system (2.7) has been studied in detail in [2] and [8].

\section{Proof of theorem 1.1}

In this section, we generalize Proposition 2.2 of [1] to the variable coefficients case. While most of places of the proof are the same to Theorem 1.1 of [16] for the constant coefficients case, we would rather give the proof in detail for the sake of completeness.

In what follows, the $C_{T}$ is used frequently to denote some positive constant that is independent of $(y, u)$ although it may change values from different contexts. We rewrite (1.1) with zero initial data by the operator $\mathcal{A}$ 
into the form:

$$
\left\{\begin{array}{l}
w_{t t}+\mathcal{A} w=0 \text { in } \Omega \times(0, \infty), \\
w(\cdot, 0)=0, w_{t}(\cdot, 0)=0 \text { in } \Omega, \\
w=u \text { on } \Gamma_{0} \times(0, \infty), \\
y=-\frac{\partial \mathcal{A}^{-1} w_{t}}{\partial \nu_{\mathcal{A}}} \text { on } \Gamma_{0} \times(0, \infty) .
\end{array}\right.
$$

By Propositions 3.2 and 3.3 of [2] (see also [23]), Theorem 1.1 is equivalent to saying that the solution to (3.1) satisfies

$$
\|y\|_{L^{2}(0, T ; U)} \leq C_{T}\|u\|_{L^{2}(0, T ; U)}, \forall u \in L^{2}(0, T ; U) .
$$

By considering $\left.u\right|_{\Gamma_{1}}=0$, we may assume without loss of generality that $\Gamma_{0}=\Gamma=\partial \Omega$. Let $z:=A^{-1} w_{t}$, where $w$ is a solution of $(3.1)$.

Since $y=-B^{*} w_{t}=-\frac{\partial \mathcal{A}^{-1} w_{t}}{\partial \nu_{\mathcal{A}}}=-\frac{\partial z}{\partial \nu_{\mathcal{A}}},(3.2)$ is equivalent to

$$
\left\|B^{*} w_{t}\right\|_{L^{2}\left(0, T ; L^{2}(\Gamma)\right)} \leq C_{T}\|u\|_{L^{2}\left(0, T ; L^{2}(\Gamma)\right)}
$$

or

$$
\left\|\frac{\partial z}{\partial \nu_{\mathcal{A}}}\right\|_{L^{2}\left(0, T ; L^{2}(\Gamma)\right)} \leq C_{T}\|u\|_{L^{2}\left(0, T ; L^{2}(\Gamma)\right)}
$$

where $z$ satisfies

$$
\left\{\begin{array}{l}
z_{t t}+\mathcal{A} z=\Upsilon u_{t} \text { in } \Omega \times(0, \infty), \\
z(\cdot, 0)=0, z_{t}(\cdot, 0)=0 \text { in } \Omega, \\
z=0 \text { on } \Gamma \times(0, \infty)
\end{array}\right.
$$

Let $\Sigma=\Gamma \times(0, T), Q=\Omega \times(0, T)$. And denote $L^{2}(\Sigma):=H^{0}(\Sigma)=L^{2}\left(0, T ; L^{2}(\Gamma)\right), H^{1}(\Sigma):=L^{2}\left(0, T ; H^{1}(\Gamma)\right) \cap$ $H^{1}\left(0, T ; L^{2}(\Gamma)\right), H^{-1}(\Sigma):=\left(H^{1}(\Sigma)\right)^{\prime}$.

The following lemma is Theorem 2.3 of [17] in the variable coefficients case, which has already been indicated in the same paper.

Lemma 3.1. Assume that

$$
F \in L^{1}\left(0, T ; H^{-1}(\Omega)\right), \Phi^{0} \in L^{2}(\Omega), \Phi^{1} \in H^{-1}(\Omega), u \in L^{2}(\Sigma)
$$

and $\widetilde{\mathcal{A}}$ is a second-order uniformly strongly elliptic operator:

$$
\widetilde{\mathcal{A}}:=-\sum_{i, j=1}^{n} \frac{\partial}{\partial x_{i}}\left(\widetilde{a}_{i j}(x) \frac{\partial}{\partial x_{j}}\right)
$$

where for some $\alpha>0$

$$
\begin{aligned}
& \widetilde{a}_{i j}(x)=\widetilde{a}_{j i}(x) \in C^{\infty}\left(\mathbb{R}^{n}\right), \sum_{i, j=1}^{n} \widetilde{a}_{i j}(x) \xi_{i} \overline{\xi_{j}} \geq \alpha|\xi|^{2}, \forall x \in \Omega, \xi=\left(\xi_{1}, \xi_{2}, \ldots, \xi_{n}\right) \in \mathbb{C}^{n}, \\
& \nu_{\widetilde{\mathcal{A}}}:=\left(\sum_{k=1}^{n} \nu_{k} \widetilde{a}_{k 1}(x), \sum_{k=1}^{n} \nu_{k} \widetilde{a}_{k 2}(x), \ldots, \sum_{k=1}^{n} \nu_{k} \widetilde{a}_{k n}(x)\right) .
\end{aligned}
$$


Then the solution $\Phi$ to the following equation

$$
\left\{\begin{array}{l}
\Phi_{t t}+\widetilde{\mathcal{A}} \Phi=F \text { in } Q \\
\Phi(\cdot, 0)=\Phi^{0}, \Phi_{t}(\cdot, 0)=\Phi^{1} \text { in } \Omega \\
\Phi=u \text { on } \Sigma
\end{array}\right.
$$

satisfies

$$
\left(\Phi, \Phi_{t}\right) \in C\left([0, T] ; L^{2}(\Omega) \times H^{-1}(\Omega)\right), \frac{\partial \Phi}{\partial \nu_{\widetilde{\mathcal{A}}}} \in H^{-1}(\Sigma) .
$$

Remark 3.1. In Lemma 3.1, one can show that $\Phi, \Phi_{t}$ and $\frac{\partial \Phi}{\partial \nu_{\tilde{\mathcal{A}}}}$ depend continuously on the given datum. Similar remarks apply to all subsequent regularity results.

Apply Lemma 3.1 to $(3.1)$ to get the regularity: $\left(w, w_{t}\right) \in C\left([0, T] ; L^{2}(\Omega) \times H^{-1}(\Omega)\right)$. This produces in turn the regularities of $z$, the solution of $(3.5)$ :

$$
\left\{\begin{array}{l}
z \in C\left([0, T] ; H_{0}^{1}(\Omega)\right), A z=w_{t} \in C\left([0, T] ; H^{-1}(\Omega)\right) \\
z_{t}=A^{-1} w_{t t}=A^{-1}[-\tilde{\mathcal{A}} w+\tilde{\mathcal{A}} \Upsilon u]=-w+\Upsilon u \in L^{2}\left(0, T ; L^{2}(\Omega)\right) .
\end{array}\right.
$$

Like Lemma 3.1, the following lemma is a direct corollary of Theorem 2.2 of [17] in the variable coefficients case.

Lemma 3.2. For the problem (3.6), if

$$
F \in L^{1}\left(0, T ; H_{0}^{1}(\Omega)\right), \Phi^{0}=\Phi^{1}=0, u=0,
$$

then $\frac{\partial \Phi}{\partial \nu_{\tilde{\mathcal{A}}}} \in H^{1}(\Sigma)$.

Lemma 3.3. Let $\Psi$ be a function defined on $\bar{Q}$. Suppose $\Psi=0$ on $\Sigma$. Then for $s=-1$ or $s=0$,

$$
\frac{\partial \Psi}{\partial \nu_{\widetilde{\mathcal{A}}}} \in H^{s}(\Sigma) \Longleftrightarrow \frac{\partial \Psi}{\partial \nu} \in H^{s}(\Sigma) .
$$

Moreover, there exists a constant $C>0$ such that

$$
C^{-1}\left\|\frac{\partial \Psi}{\partial \nu}\right\|_{H^{s}(\Sigma)} \leq\left\|\frac{\partial \Psi}{\partial \nu_{\tilde{\mathcal{A}}}}\right\|_{H^{s}(\Sigma)} \leq C\left\|\frac{\partial \Psi}{\partial \nu}\right\|_{H^{s}(\Sigma)}
$$

Proof. Since $\Psi=0$ on $\Sigma$, it has $\frac{\partial \Psi}{\partial x_{j}}=\nu_{j} \frac{\partial \Psi}{\partial \nu}$ on $\Sigma$ for any $1 \leq j \leq n$, and so $\frac{\partial \Psi}{\partial \nu_{\tilde{\mathcal{A}}}}=\sum_{i, j=1}^{n} \widetilde{a}_{i j} \nu_{i} \nu_{j} \frac{\partial \Psi}{\partial \nu}$ on $\Sigma$. By assumption, we may assume that

$$
C^{-1} \leq \sup _{x \in \bar{\Omega}} \sum_{i, j=1}^{n} \widetilde{a}_{i j} \nu_{i} \nu_{j} \leq C
$$

for some $C>0$. We only show that $\frac{\partial \Psi}{\partial \nu_{\tilde{\mathcal{A}}}} \in H^{-1}(\Sigma) \Longrightarrow \frac{\partial \Psi}{\partial \nu} \in H^{-1}(\Sigma)$ and $\left\|\frac{\partial \Psi}{\partial \nu}\right\|_{H^{-1}(\Sigma)} \leq C\left\|\frac{\partial \Psi}{\partial \nu_{\tilde{\mathcal{A}}}}\right\|_{H^{-1}(\Sigma)}$ since the proofs for other cases are similar. Now, for any $h \in H_{0}^{1}(\Sigma)$, it has

$$
\begin{aligned}
\left|\int_{\Sigma} \frac{\partial \Psi}{\partial \nu} h \mathrm{~d} \Sigma\right| & =\left|\int_{\Sigma} \frac{\partial \Psi}{\partial \nu_{\widetilde{\mathcal{A}}}} \frac{h}{\sum_{i, j=1}^{n} \widetilde{a}_{i j} \nu_{i} \nu_{j}} \mathrm{~d} \Sigma\right| \leq\left\|\frac{\partial \Psi}{\partial \nu_{\widetilde{\mathcal{A}}}}\right\|_{H^{-1}(\Sigma)} \cdot\left\|\frac{h}{\sum_{i, j=1}^{n} \widetilde{a}_{i j} \nu_{i} \nu_{j}}\right\|_{H_{0}^{1}(\Sigma)} \\
& \leq C\left\|\frac{\partial \Psi}{\partial \nu_{\widetilde{\mathcal{A}}}}\right\|_{H^{-1}(\Sigma)} \cdot\|h\|_{H_{0}^{1}(\Sigma)} \cdot
\end{aligned}
$$

This yields $\left\|\frac{\partial \Psi}{\partial \nu}\right\|_{H^{-1}(\Sigma)} \leq C\left\|\frac{\partial \Psi}{\partial \nu_{\tilde{A}}}\right\|_{H^{-1}(\Sigma)}$. The proof is complete. 
Proof of Theorem 1.1. The proof will be split into four steps.

Step 1. Let $u \in L^{2}(\Sigma)$. Then by Lemma 3.1, the solution to (3.1) satisfies

$$
\left(w, w_{t}\right) \in C\left([0, T] ; L^{2}(\Omega) \times H^{-1}(\Omega)\right),\left.\frac{\partial w}{\partial \nu_{\mathcal{A}}}\right|_{\Sigma} \in H^{-1}(\Sigma) .
$$

Since $\Upsilon u \in L^{2}\left(0, T ; H^{1 / 2}(\Omega)\right)$, it follows that [13]

$$
\frac{\partial}{\partial \nu_{\mathcal{A}}} \Upsilon u \in L^{2}\left(0, T ; H^{-1}(\Gamma)\right) \subset H^{-1}(\Sigma)
$$

Substituting (3.8) and (3.9) into (3.7) yields

$$
\frac{\partial z_{t}}{\partial \nu_{\mathcal{A}}}=-\frac{\partial w}{\partial \nu_{\mathcal{A}}}+\frac{\partial}{\partial \nu_{\mathcal{A}}} \Upsilon u \in H^{-1}(\Sigma) .
$$

Since $z=0$ on $\Sigma$, so does $z_{t}$ on $\Sigma$. Therefore, it follows from Lemma 3.3 that

$$
\frac{\partial z_{t}}{\partial \nu} \in H^{-1}(\Sigma)
$$

Step 2. Like the case of constant coefficients in [11], we can use the transform of geodesic normal coordinates to change locally $\Omega$ and $\Gamma$ to $\widehat{\Omega}:=\left\{(x, y) \in \mathbb{R}^{n}, x>0, y \in \mathbb{R}^{n-1}\right\}$ and $\widehat{\Gamma}:=\left\{(x, y) \in \mathbb{R}^{n}, x=0, y \in \mathbb{R}^{n-1}\right\}$. Under such a change of coordinates, the operator $\mathcal{A}$ is changed locally to $\widehat{\mathcal{A}}:=D_{x}^{2}+r(x, y) D_{y}^{2}-$ lot ([18] or Cor. C.5.3 of [12]), where "lot" denotes the first-order differential operators and $r(x, y) D_{y}^{2}$ stands for the second-order tangential strongly elliptic operator in $y$ variable. Denote by $\widehat{w}$ for $w$, the solution of (3.1), and by $\widehat{u}$ for $u$ under this change of coordinates. Since $\widehat{w}$ has zero initial data, one can also extend $\widehat{w}(t)$ to be zero for $t<0$. Let $\phi \in C_{0}^{\infty}(\mathbb{R}),|\phi| \leq 1$, be a smooth cutoff function in $\mathbb{R}$ with $\phi(t)=0$ for $t \geq(3 / 2) T$ and $\phi(t)=1$ while $t \in[0, T]$ and put

Then $v$ satisfies

$$
v:=\widehat{w} \phi
$$

$$
\left\{\begin{array}{l}
v_{t t}=-\widehat{\mathcal{A}} v=-\mathcal{A}_{0} v+\operatorname{lot}(\widehat{w}) \text { in } \widehat{\Omega} \times(0, \infty), \\
v(\cdot, 0)=v_{t}(\cdot, 0)=0 \text { in } \widehat{\Omega}, \\
v=\phi \widehat{u} \text { on } \widehat{\Gamma} \times(0, \infty), \\
\operatorname{supp}(v) \subset[0,(3 / 2) T],
\end{array}\right.
$$

where $\mathcal{A}_{0}:=D_{x}^{2}+r(x, y) D_{y}^{2}$ is the principal part of $\widehat{\mathcal{A}}$. Denote $\widehat{\Sigma}:=\widehat{\Gamma} \times(0, T)$.

Now, decompose $v=\varphi+\psi$, where $\varphi, \psi$ satisfy (3.11) and (3.13) below, respectively.

By Lemma 3.1, the solution $\varphi$ to the following equation

$$
\left\{\begin{array}{l}
\varphi_{t t}+\mathcal{A}_{0} \varphi=0 \text { in } \widehat{\Omega} \times(0, \infty) \\
\varphi(\cdot, 0)=\varphi_{t}(\cdot, 0)=0 \text { in } \widehat{\Omega} \\
\varphi=\phi \widehat{u} \text { on } \widehat{\Gamma} \times(0, \infty)
\end{array}\right.
$$

satisfies

$\psi$ satisfies the following equation

$$
\left(\varphi, \varphi_{t}\right) \in C\left([0, T] ; L^{2}(\widehat{\Omega}) \times H^{-1}(\widehat{\Omega})\right)
$$

$$
\left\{\begin{array}{l}
\psi_{t t}+\mathcal{A}_{0} \psi=f \text { in } \widehat{\Omega} \times(0, \infty) \\
\psi(\cdot, 0)=\psi_{t}(\cdot, 0)=0 \text { in } \widehat{\Omega} \\
\psi=0 \text { on } \widehat{\Gamma} \times(0, \infty)
\end{array}\right.
$$


where $f=\operatorname{lot}(\widehat{w})$. Recalling that $w \in C\left([0, T] ; L^{2}(\Omega)\right)$ by $(3.8)$ and so $\widehat{w} \in C\left([0, T] ; L^{2}(\widehat{\Omega})\right)$, we obtain, by Lemma 3.1, that

$$
f \in C\left([0, T] ; H^{-1}(\widehat{\Omega})\right) \text { and hence }\left(\psi, \psi_{t}\right) \in C\left([0, T] ; L^{2}(\widehat{\Omega}) \times H^{-1}(\widehat{\Omega})\right) .
$$

This together with (3.12) gives

$$
\left(v, v_{t}\right) \in C\left([0, T] ; L^{2}(\widehat{\Omega}) \times H^{-1}(\widehat{\Omega})\right) .
$$

Step 3. We show that for the nonhomogeneous problem (3.13), the map

$$
\widehat{u} \mapsto B^{*} \psi_{t} \text { : is continuous from } L^{2}(\widehat{\Sigma}) \text { to } L^{2}(\widehat{\Sigma}) \text {. }
$$

Indeed, since the map $\widehat{u} \mapsto f=\operatorname{lot}(\widehat{w})$ is continuous from $L^{2}(\widehat{\Sigma})$ to $L^{2}\left(0, T ; H^{-1}(\widehat{\Omega})\right)$, it suffices to show that

$$
f \mapsto B^{*} \psi_{t}: \text { is continuous from } L^{2}\left(0, T ; H^{-1}(\widehat{\Omega})\right) \text { to } L^{2}(\widehat{\Sigma}) .
$$

Apply $\mathcal{A}_{0}^{-1}$ to $(3.13)$ to give

$$
\left\{\begin{array}{l}
\Psi_{t t}+\mathcal{A}_{0} \Psi=\mathcal{A}_{0}^{-1} f \text { in } \widehat{\Omega} \times(0, \infty), \\
\Psi(\cdot, 0)=\Psi_{t}(\cdot, 0)=0 \text { in } \widehat{\Omega}, \\
\Psi=0 \text { on } \widehat{\Gamma} \times(0, \infty),
\end{array}\right.
$$

where $\Psi:=\mathcal{A}_{0}^{-1} \psi$ satisfies, by (3.14), that

$$
\Psi \in H^{2}(\widehat{\Omega}) \cap H_{0}^{1}(\widehat{\Omega}), \mathcal{A}_{0}^{-1} f \in L^{2}\left(0, T ; H_{0}^{1}(\widehat{\Omega})\right), \mathcal{A}_{0}^{-1} \psi_{t} \in C\left([0, T] ; H_{0}^{1}(\widehat{\Omega})\right) .
$$

Apply Lemma 3.2 to problem (3.17) to obtain

$$
\frac{\partial \Psi}{\partial \nu_{\mathcal{A}_{0}}} \in H^{1}(\widehat{\Sigma}) \text { and so } \frac{\partial \Psi_{t}}{\partial \nu_{\mathcal{A}_{0}}} \in L^{2}(\widehat{\Sigma})
$$

Finally, by (2.6), $B^{*} \psi_{t}=B^{*} \mathcal{A}_{0} \mathcal{A}_{0}^{-1} \psi_{t}=B^{*} \mathcal{A}_{0} \Psi_{t}=\frac{\partial \Psi_{t}}{\partial \nu_{\mathcal{A}_{0}}}$, it follows from Remark 3.1 that $\mathcal{A}_{0}^{-1} f \mapsto \frac{\partial \Psi_{t}}{\partial \nu_{\mathcal{A}_{0}}}=$ $B^{*} \psi_{t}$ is continuous from $L^{2}\left(0, T ; H_{0}^{1}(\widehat{\Omega})\right)$ to $L^{2}(\widehat{\Sigma})$. (3.16) then follows from a trivial fact that $f \mapsto \mathcal{A}_{0}^{-1} f$ is continuous from $L^{2}\left(0, T ; H^{-1}(\widehat{\Omega})\right)$ to $L^{2}\left(0, T ; H_{0}^{1}(\widehat{\Omega})\right)$.

Step 4. The proof will be accomplished if we can show that for problem (3.11), the map

$$
\widehat{u} \mapsto B^{*} \varphi_{t} \text { : is continuous from } L^{2}(\widehat{\Sigma}) \text { to } L^{2}(\widehat{\Sigma}) .
$$

Comparing problem (3.1) with problem (3.11) and noticing the equivalences of (3.2), (3.3) and (3.4), we find that (3.18) is equivalent to

$$
\left\|\frac{\partial \widehat{z}}{\partial \nu_{\mathcal{A}_{0}}}\right\|_{L^{2}(\widehat{\Sigma})} \leq C_{T}\|\widehat{u}\|_{L^{2}(\widehat{\Sigma})}, \text { where } \widehat{z}:=\mathcal{A}_{0}^{-1} \varphi_{t}
$$

Let $\mathcal{X}(x, y, t) \in S^{0}(\widehat{\Omega} \times \mathbb{R})$ be a pseudodifferential operator with smooth symbol of localization $\chi(x, y, t, \eta, \sigma)$ supported in the elliptic sector of $\square:=D_{t}^{2}-D_{x}^{2}-r(x, y) D_{y}^{2}$, where the principal part of the D'Alambertian is written in local coordinates. Here the dual variables $\eta \in \mathbb{R}^{n-1}, \sigma \in \mathbb{R}$ correspond to the Fourier's variables of $y \mapsto i \eta, t \mapsto i \sigma$. Suppose $\operatorname{supp}(\chi) \subset\left\{(x, y, t, \eta, \sigma) \in \widehat{\Omega} \times \mathbb{R} \times \mathbb{R}^{n-1} \times \mathbb{R},|\sigma| \leq C_{1}|\eta|\right\}$, and $\operatorname{supp}(1-\chi) \subset$ $\left\{(x, y, t, \eta, \sigma) \in \widehat{\Omega} \times \mathbb{R} \times \mathbb{R}^{n-1} \times \mathbb{R},|\sigma| \geq C_{2}|\eta|\right\}$, where $0<C_{2}<C_{1}$ are constants. We show that

$$
(I-\mathcal{X}) \frac{\partial \widehat{z}}{\partial \nu} \in L^{2}(\widehat{\Sigma})
$$


The trick comes from the application of (3.10). Actually, by (3.10), it has

$$
\left(1+\sigma^{2}+|\eta|^{2}\right)^{-\frac{1}{2}}(i \sigma) \frac{\partial \tilde{z}}{\partial \nu} \in L^{2}\left(\mathbb{R}_{\eta, \sigma}^{n}\right)
$$

where $\tilde{z}$ is the partial Fourier transform of $\widehat{z}$ respect to $(y, t)$. Hence

$$
\int_{\mathbb{R}^{n}} \frac{\sigma^{2}}{1+\sigma^{2}+|\eta|^{2}}\left|\frac{\partial \tilde{z}}{\partial \nu}\right|^{2} \mathrm{~d} \eta \mathrm{d} \sigma<+\infty .
$$

Since when $|\sigma| \geq C_{2}|\eta|,|\eta| \geq 1$, it has

$$
\frac{C_{2}^{2}}{C_{2}^{2}+2} \leq \frac{C_{2}^{2}}{\frac{1}{|\eta|^{2}}+C_{2}^{2}+1}=\frac{C_{2}^{2}|\eta|^{2}}{1+|\eta|^{2} C_{2}^{2}+|\eta|^{2}} \leq \frac{\sigma^{2}}{1+\sigma^{2}+|\eta|^{2}},
$$

hence

Therefore

$$
\begin{aligned}
\int_{|\sigma| \geq C_{2}|\eta|}\left|\frac{\partial \tilde{z}}{\partial \nu}\right|^{2} \mathrm{~d} \eta \mathrm{d} \sigma & \leq \int_{|\sigma| \geq C_{2}|\eta|} \frac{2+C_{2}^{2}}{C_{2}^{2}} \frac{\sigma^{2}}{1+\sigma^{2}+|\eta|^{2}}\left|\frac{\partial \tilde{z}}{\partial \nu}\right|^{2} \mathrm{~d} \eta \mathrm{d} \sigma \\
& \leq \frac{2+C_{2}^{2}}{C_{2}^{2}} \int_{\mathbb{R}^{n}} \frac{\sigma^{2}}{1+\sigma^{2}+|\eta|^{2}}\left|\frac{\partial \tilde{z}}{\partial \nu}\right|^{2} \mathrm{~d} \eta \mathrm{d} \sigma<\infty
\end{aligned}
$$

$$
\begin{aligned}
\int_{\mathbb{R}^{n}}|1-\chi|^{2}\left|\frac{\partial \tilde{z}}{\partial \nu}\right|^{2} \mathrm{~d} \eta \mathrm{d} \sigma & =\int_{\operatorname{supp}(1-\chi)}|1-\chi|^{2}\left|\frac{\partial \tilde{z}}{\partial \nu}\right|^{2} \mathrm{~d} \eta \mathrm{d} \sigma \\
& \leq \int_{|\sigma| \geq C_{2}|\eta|}\left|\frac{\partial \tilde{z}}{\partial \nu}\right|^{2} \mathrm{~d} \eta \mathrm{d} \sigma<\infty
\end{aligned}
$$

Thus (3.20) is valid.

Now, we need to show that $\mathcal{X} \frac{\partial \widehat{z}}{\partial \nu} \in L^{2}(\widehat{\Sigma})$. This is a little bit easy due to its ellipticity. Returning to problem (3.11) for $\varphi$, rewritten as $\square \varphi=0$ and applying $\mathcal{X}$, we will see that the variable $\mathcal{X} \varphi$ satisfies

$$
\left\{\begin{array}{l}
\square \mathcal{X} \varphi=-[\mathcal{X}, \square] \varphi \in H^{-1}(\widetilde{Q}) \\
\left.\mathcal{X} \varphi\right|_{\partial \widetilde{Q}} \in L^{2}(\partial \widetilde{Q})
\end{array}\right.
$$

where and henceforth we take for $\widetilde{Q}$ an extend cylinder based on $\widehat{\Omega} \times[-T, 2 T]$ and denote $\widetilde{\Sigma}:=\widehat{\Gamma} \times[-T, 2 T]$. Indeed, by the fact that $[\mathcal{X}, \square] \in S^{1}(\widetilde{Q})$ and the priori regularity in $(3.12)$, we have $[\mathcal{X}, \square] \varphi \in H^{-1}(\widetilde{Q})$.

Furthermore, $\left.\mathcal{X} \varphi\right|_{\widetilde{\Sigma}}=\mathcal{X} \phi \widehat{u} \in L^{2}(\widetilde{\Sigma})$. And by the pseudolocal property of pseudodifferential operators and the fact that $\operatorname{supp}(\varphi) \subset[0,(3 / 2) T]$, we have $(\mathcal{X} \varphi)(2 T, \cdot) \in C^{\infty}(\widehat{\Omega})$ and $(\mathcal{X} \varphi)(-T, \cdot) \in C^{\infty}(\widehat{\Omega})$. This yields the boundary condition $\left.\mathcal{X} \varphi\right|_{\partial \widetilde{Q}} \in L^{2}(\partial \widetilde{Q})$ in (3.21). Since $\square \mathcal{X}$ is a pseudodifferential elliptic operator, apply the classical elliptic theory to the elliptic problem (3.21) to yield

$$
\mathcal{X} \varphi \in H^{1 / 2}(\widetilde{Q})+H^{1}(\widetilde{Q})=H^{1 / 2}(\widetilde{Q}),
$$

where the first term in the middle of (3.22) is due to the boundary regularity of (3.21), and the second term is due to the interior regularity. Next, we return to the elliptic problem

$$
\left\{\begin{array}{l}
\mathcal{A} z=w_{t} \text { in } Q \\
\left.z\right|_{\Sigma}=0
\end{array}\right.
$$


from (3.7). Since $\widehat{z}:=\mathcal{A}_{0}^{-1} \varphi_{t}$ in $\widetilde{Q}$, the counterpart of the above elliptic problem in the half-space $\widetilde{Q}$ is (locally)

$$
\left\{\begin{array}{l}
\mathcal{A}_{0} \widehat{z}=\varphi_{t} \text { in } \widetilde{Q} \\
\left.\widehat{z}\right|_{\widetilde{\Sigma}}=0
\end{array}\right.
$$

Apply $\mathcal{X}$ to above problem to yield

$$
\mathcal{A}_{0} \mathcal{X} \widehat{z}=\mathcal{X} \varphi_{t}+\left[\mathcal{A}_{0}, \mathcal{X}\right] \widehat{z}=\frac{\mathrm{d}}{\mathrm{d} t} \mathcal{X} \varphi-\left[\frac{\mathrm{d}}{\mathrm{d} t}, \mathcal{X}\right] \varphi+\left[\mathcal{A}_{0}, \mathcal{X}\right] \widehat{z}
$$

Notice that $\left[\mathcal{A}_{0}, \mathcal{X}\right] \in S^{1}(\widetilde{Q})$ and $\left[\frac{\mathrm{d}}{\mathrm{d} t}, \mathcal{X}\right] \in S^{0}(\widetilde{Q})$. By the priori regularity in $(3.12)$ for $\varphi$ and in $(3.7)$ for $z$, we conclude that

$$
-\left[\frac{\mathrm{d}}{\mathrm{d} t}, \mathcal{X}\right] \varphi+\left[\mathcal{A}_{0}, \mathcal{X}\right] \widehat{z} \in L^{2}(\widetilde{Q}) .
$$

Moreover, by virtue of $(3.22), \mathcal{X} \varphi \in H_{\left(\frac{1}{2}, \frac{1}{2}\right)}(\widetilde{Q}) \subset H_{\left(0, \frac{1}{2}\right)}(\widetilde{Q})$, where we have used the anisotropic Hörmander's spaces of [12] on page 477 . In the space $H_{(m, s)}(\widetilde{Q}), m$ is the order in the normal direction to the plane $x=0$ (which plays a distinguished role) and $(m+s)$ is the order in the tangential direction in $t$ and $y$. Since $\frac{\mathrm{d}}{\mathrm{d} t}$ is a first order differential operator in the tangential direction, $\frac{\mathrm{d}}{\mathrm{d} t} \mathcal{X} \varphi \in H_{\left(0,-\frac{1}{2}\right)}(\widetilde{Q}) \subset H_{\left(-\frac{1}{2}, 0\right)}(\tilde{Q})=H^{-\frac{1}{2}}(\widetilde{Q})$. By (3.23), we are led to solving the following problem

$$
\left\{\begin{array}{l}
\mathcal{A}_{0} \mathcal{X} \widehat{z} \in H^{-1 / 2}(\widetilde{Q})+L^{2}(\widetilde{Q}) \subset H^{-1 / 2}(\widetilde{Q}) \\
\left.(\mathcal{X} \widehat{z})\right|_{\widetilde{\Sigma}}=0
\end{array}\right.
$$

Since $\mathcal{A}_{0} \mathcal{X}$ is elliptic in $\widetilde{Q}$, by the classical elliptic regularity again, we obtain

$$
\mathcal{X} \widehat{z} \in H^{3 / 2}(\widetilde{Q}), \frac{\partial}{\partial \nu} \mathcal{X} \widehat{z} \in L^{2}(\widetilde{\Sigma})
$$

Combining (3.24) and (3.20) yields

$$
\frac{\partial \widehat{z}}{\partial \nu}=(I-\mathcal{X}) \frac{\partial \widehat{z}}{\partial \nu}+\mathcal{X} \frac{\partial \widehat{z}}{\partial \nu} \in L^{2}(\widehat{\Sigma})
$$

Finally, since $\widehat{z}=0$ on $\widehat{\Sigma}$, it follows from Lemma 3.3 that

$$
\frac{\partial \widehat{z}}{\partial \nu_{\mathcal{A}_{0}}} \in L^{2}(\widehat{\Sigma})
$$

proving (3.19). The proof is complete.

\section{Proof of Theorem 1.2}

Although here we consider the variable coefficients case, the proof below is much simpler than the one in [11] for the case of constant coefficients. This is due to the use of multiplier method on Riemannian manifolds, which was an effective tool introduced in [24]. In the case of constant coefficients, the multipliers are reduced to the usual ones in Euclidean spaces.

Notice the hypothesis (1.2) and set

$$
A(x):=\left(a_{i j}(x)\right), G(x):=\left(g_{i j}(x)\right)=A(x)^{-1}, \mathcal{G}(x):=\operatorname{det}\left(g_{i j}(x)\right) .
$$


Let $\mathbb{R}^{n}$ be the usual Euclidean space. For each $x=\left(x_{1}, x_{2}, \ldots, x_{n}\right) \in \mathbb{R}^{n}$, define the inner product and norm over the tangent space $\mathbb{R}_{x}^{n}=\mathbb{R}^{n}$ by

$$
\begin{aligned}
& g(X, Y):=\langle X, Y\rangle_{g}=\sum_{i, j=1}^{n} g_{i j}(x) \alpha_{i} \beta_{j} \\
& |X|_{g}:=\langle X, X\rangle_{g}^{1 / 2}, \forall X=\sum_{i=1}^{n} \alpha_{i} \frac{\partial}{\partial x_{i}}, Y=\sum_{i=1}^{n} \beta_{i} \frac{\partial}{\partial x_{i}} \in \mathbb{R}_{x}^{n} .
\end{aligned}
$$

It is easily checked that $\left(\mathbb{R}^{n}, g\right)$ is a Riemannian manifold with Riemannian metric $g$. Denote by $D$ the LeviCivita connection with respect to $g$. Let $H$ be a vector field on $\left(\mathbb{R}^{n}, g\right)$. Then for each $x \in \mathbb{R}^{n}$, the covariant differential $D H$ of $H$ determines a bilinear form on $\mathbb{R}_{x}^{n} \times \mathbb{R}_{x}^{n}$ :

$$
D H(X, Y)=\left\langle D_{X} H, Y\right\rangle_{g}, \forall X, Y \in \mathbb{R}_{x}^{n},
$$

where $D_{X} H$ stands for the covariant derivative of vector field $H$ with respect to $X$.

For any $f \in C^{2}\left(\mathbb{R}^{n}\right), X=\sum_{i=1}^{n} \alpha_{i} \frac{\partial}{\partial x_{i}}$, denote

$$
\begin{aligned}
& \nabla_{g} f:=\sum_{i, j=1}^{n} \frac{\partial f}{\partial x_{i}} a_{i j}(x) \frac{\partial}{\partial x_{j}}, \operatorname{div}_{g} X:=\sum_{i=1}^{n} \frac{1}{\sqrt{\mathcal{G}(x)}} \frac{\partial}{\partial x_{i}}\left(\sqrt{\mathcal{G}(x)} \alpha_{i}(x)\right) \\
& \Delta_{g} f:=\sum_{i, j=1}^{n} \frac{1}{\sqrt{\mathcal{G}(x)}} \frac{\partial}{\partial x_{i}}\left(\sqrt{\mathcal{G}(x)} a_{i j}(x) \frac{\partial f}{\partial x_{j}}\right)=-\mathcal{A} f+\mathcal{T} f, \mathcal{T}:=\sum_{i, j=1}^{n} \frac{a_{i j}(x)}{\sqrt{\mathcal{G}(x)}} \frac{\partial \sqrt{\mathcal{G}(x)}}{\partial x_{i}} \frac{\partial}{\partial x_{j}}
\end{aligned}
$$

Now, it follows from the Appendix of [8] that the transfer function of the system (2.7) is

$$
H(\lambda)=\lambda B^{*}\left(\lambda^{2}+\tilde{A}\right)^{-1} B
$$

where $\tilde{A}, B$ and $B^{*}$ are given by (2.3), (2.4) and (2.6), respectively. Moreover, from the well-posedness claimed by Theorem 1.1, it follows that there are constants $M, \beta>0$ such that ([6])

$$
\sup _{\operatorname{Re} \lambda \geq \beta}\|H(\lambda)\|_{\mathcal{L}(U)}=M<\infty .
$$

Proposition 4.1. Theorem 1.2 is valid if for any $u \in C_{0}^{\infty}\left(\Gamma_{0}\right)$, the solution $w$ to the following equation

$$
\left\{\begin{array}{l}
\lambda^{2} w(x)=\sum_{i, j=1}^{n} \frac{\partial}{\partial x_{i}}\left(a_{i j}(x) \frac{\partial w}{\partial x_{j}}(x)\right)=-\mathcal{A} w(x), x \in \Omega \\
w(x)=0, x \in \Gamma_{1} \\
w(x)=u(x), x \in \Gamma_{0}
\end{array}\right.
$$

satisfies

$$
\left.\left.\lim _{\lambda \in \mathbb{R}, \lambda \rightarrow+\infty} \int_{\Gamma_{0}}\left|\frac{1}{\lambda} \frac{\partial w(x)}{\partial \nu_{\mathcal{A}}}-\right| \nu_{\mathcal{A}}\right|_{g} u(x)\right|^{2} \mathrm{~d} x=0 .
$$

Proof. It was shown in [21] that in the frequency domain, (1.4) is equivalent to

$$
\lim _{\lambda \in \mathbb{R}, \lambda \rightarrow+\infty} H(\lambda) u=\left|\nu_{\mathcal{A}}\right|_{g} u \text { in the strong topology of } U \text { for any } u \in U,
$$


where $H(\lambda)$ is given by (4.1). Due to (4.2) and a density argument, it suffices to show that (4.4) is satisfied for all $u \in C_{0}^{\infty}\left(\Gamma_{0}\right)$.

Now assume that $u \in C_{0}^{\infty}\left(\Gamma_{0}\right)$, and put

$$
w(x)=-\left(\left(\lambda^{2}+\tilde{A}\right)^{-1} B u\right)(x) .
$$

Then $w$ satisfies (4.3) and

$$
(H(\lambda) u)(x)=-\lambda \frac{\partial\left(\mathcal{A}^{-1} w\right)}{\partial \nu_{\mathcal{A}}}(x), \forall x \in \Gamma_{0} .
$$

Take a function $v \in H^{2}(\Omega)$ to satisfy

$$
\left\{\begin{array}{l}
\sum_{i, j=1}^{n} \frac{\partial}{\partial x_{i}}\left(a_{i j}(x) \frac{\partial v(x)}{\partial x_{j}}\right)=0, x \in \Omega \\
v(x)=0, x \in \Gamma_{1} ; v(x)=u(x), x \in \Gamma_{0}
\end{array}\right.
$$

Then (4.3) can be written as

$$
\left\{\begin{array}{l}
\lambda^{2} w(x)-\sum_{i, j=1}^{n} \frac{\partial}{\partial x_{i}}\left(a_{i j}(x) \frac{\partial}{\partial x_{j}}(w(x)-v(x))\right)=0, x \in \Omega \\
\left.(w-v)\right|_{\partial \Omega}=0
\end{array}\right.
$$

or

$$
-\lambda^{2}\left(\mathcal{A}^{-1} w\right)(x)=w(x)-v(x)
$$

So (4.5) becomes

$$
(H(\lambda) u)(x)=\frac{1}{\lambda} \frac{\partial w(x)}{\partial \nu_{\mathcal{A}}}-\frac{1}{\lambda} \frac{\partial v(x)}{\partial \nu_{\mathcal{A}}}
$$

Since $\frac{\partial v(x)}{\partial \nu_{\mathcal{A}}}$ is independent of $\lambda$, the required result then follows from (4.6) and (4.4).

The following lemma, which may be useful for other purposes, is the generalization of Lemma 2.1 in [14], p. 18, which refers to Euclidean spaces, to the Riemannian manifold $(\bar{\Omega}, g)$.

Lemma 4.1. Suppose $\partial \Omega$ is of class $C^{k}, k \geq 1$. Assume (1.2) and (1.3). Then there exists a vector field $N: \bar{\Omega} \rightarrow \mathbb{R}^{n}$ of class $C^{k-1}$ such that

$$
N(x)=\mu(x), x \in \partial \Omega ;|N|_{g} \leq 1, x \in \Omega
$$

where $\mu:=\frac{\nu_{\mathcal{A}}}{\left|\nu_{\mathcal{A}}\right|_{g}}$ is the unit normal of $\partial \Omega$ pointing towards the exterior of $\Omega$ in terms of the Riemannian metric $g$.

Proof. Since $\partial \Omega$ is of class $C^{k}, k \geq 1$, for every fixed $x^{0} \in \partial \Omega$, there is an open neighborhood $V$ of $x^{0}$ in $\mathbb{R}^{n}$ and a function $\phi: V \rightarrow \mathbb{R}$ of class $C^{k}$ such that

$$
\nabla_{g} \phi(x) \neq 0, \forall x \in V \text { and } \phi(x)=0 \text { iff } x \in V \cap \partial \Omega
$$

Replacing $\phi$ by $-\phi$ if needed, we may assume that

$$
\left\langle\mu\left(x^{0}\right), \nabla_{g} \phi\left(x^{0}\right)\right\rangle_{g}>0
$$


Then the function $\psi:=\nabla_{g} \phi /\left|\nabla_{g} \phi\right|_{g}: V \rightarrow \mathbb{R}^{n}$ is of class $C^{k-1}$. We show that $\psi=\mu$ on $V \cap \partial \Omega$. In fact, since $\phi=0$ on $V \cap \partial \Omega$, we have

Hence

$$
\frac{\partial \phi}{\partial x_{j}}=\nu_{j}\left(\frac{\partial \phi}{\partial \nu}\right) \text { on } V \cap \partial \Omega, j=1,2, \ldots, n \text {. }
$$

$$
\nabla_{g} \phi=\sum_{i, j=1}^{n} \frac{\partial \phi}{\partial x_{i}} a_{i j} \frac{\partial}{\partial x_{j}}=\sum_{i, j=1}^{n} \nu_{i}\left(\frac{\partial \phi}{\partial \nu}\right) a_{i j} \frac{\partial}{\partial x_{j}}=\frac{\partial \phi}{\partial \nu}\left(\sum_{i, j=1}^{n} \nu_{i} a_{i j} \frac{\partial}{\partial x_{j}}\right)=\frac{\partial \phi}{\partial \nu} \cdot \nu_{\mathcal{A}}
$$

which implies that $\psi, \nabla_{g} \phi, \nu_{\mathcal{A}}$ and $\mu$ are parallel to each other on $V \cap \partial \Omega$. This together with $|\psi|_{g}=|\mu|_{g}=1$ shows that $\psi=\mu$ on $V \cap \partial \Omega$.

Since $\Omega$ is bounded, $\partial \Omega$ is compact in $\mathbb{R}^{n}$; therefore $\partial \Omega$ can be covered with a finite number of neighborhoods $V_{1}, V_{2}, \ldots, V_{m}$. Each of them plays the role of $V$ in the earlier reasoning. Denoting by $\psi_{i}, i=1,2, \ldots, m$ the corresponding functions of $V_{i}$, we have

$$
\partial \Omega \subset V_{1} \cup V_{2} \ldots \cup V_{m}
$$

and

Fix an open set $V_{0} \subset \mathbb{R}^{n}$ such that

$$
\psi_{i}=\mu \text { on } V_{i} \cap \partial \Omega, i=1,2, \ldots, m
$$

$$
\bar{\Omega} \subset V_{0} \cup V_{1} \cup \cdots \cup V_{m}, V_{0} \cap \partial \Omega=\emptyset
$$

and define $\psi_{0}: V_{0} \rightarrow \mathbb{R}^{n}$ by $\psi_{0}(x)=0$ for all $x \in V_{0}$.

Let $\theta_{0}, \theta_{1}, \ldots, \theta_{m}$ be a partition of unity of class $C^{k}$, corresponding to the covering $V_{0}, V_{1}, \ldots, V_{m}$ of $\bar{\Omega}$ :

$$
\theta_{i} \in C_{0}^{k}\left(V_{i}\right) \text { and } 0 \leq \theta_{i} \leq 1, i=0,1, \ldots, m
$$

and

It is obvious that

$$
\theta_{0}+\theta_{1}+\cdots+\theta_{m}=1 \text { on } \bar{\Omega}
$$

is the required vector field.

$$
N:=\left.\left(\sum_{i=0}^{m} \theta_{i} \psi_{i}\right)\right|_{\bar{\Omega}}
$$

Proof of Theorem 1.2. We define $\tilde{u} \in C^{\infty}(\Gamma)$ as the extension of $u$, i.e. $\tilde{u}=u$ on $\Gamma_{0}$ and $\tilde{u}=0$ on $\Gamma_{1}$. Set

$$
F(\lambda) \tilde{u}:=\frac{1}{\lambda} \frac{\partial w}{\partial \mu}, \quad x \in \Gamma
$$

where $w$ satisfies (4.3) with $u \in C_{0}^{\infty}\left(\Gamma_{0}\right) . \quad \mu=\frac{\nu_{\mathcal{A}}}{\left|\nu_{\mathcal{A}}\right|_{g}}$ and $\frac{\partial}{\partial \mu}$ is defined similarly as $\frac{\partial}{\partial \nu_{\mathcal{A}}}$ in (1.3): $\frac{\partial}{\partial \mu}:=$ $\sum_{i=1}^{n} \mu_{i} \frac{\partial}{\partial x_{i}}=\frac{1}{\left|\nu_{\mathcal{A}}\right|_{g}} \cdot \frac{\partial}{\partial \nu_{\mathcal{A}}}$. The proof will be accomplished if we can show that

$$
\lim _{\lambda \in \mathbb{R}, \lambda \rightarrow \infty} F(\lambda) \tilde{u}=\tilde{u} \text { on } L^{2}(\Gamma),
$$

which will be split into two steps.

Step 1. Let the vector field $N$ be given by (4.7). Since $\tilde{u} \in C^{\infty}(\Gamma)$, the solution $w$ to (4.3) belongs to $C^{\infty}(\Omega)$ and hence $N(\bar{w}) \in \mathbb{C}$. Multiply the both sides of the first equation of $(4.3)$ by $N(\bar{w})$ and integrate by parts. By formulae for divergence on the Riemannian manifold $(\bar{\Omega}, g)$ :

$$
\operatorname{div}_{g}\left(|w|^{2} N\right)=N\left(|w|^{2}\right)+|w|^{2} \operatorname{div}_{g}(N)
$$


and

we obtain

$$
\int_{\Omega} \operatorname{div}_{g}\left(|w|^{2} N\right) \mathrm{d} x=\int_{\Gamma}\left\langle|w|^{2} N, \mu\right\rangle_{g} \mathrm{~d} \Gamma
$$

$$
\begin{aligned}
\operatorname{LHS}=\operatorname{Re}\left(\lambda^{2} \int_{\Omega} w N(\bar{w}) \mathrm{d} x\right) & =\frac{\lambda^{2}}{2} \int_{\Omega} N\left(|w|^{2}\right) \mathrm{d} x=\frac{\lambda^{2}}{2} \int_{\Omega}\left[\operatorname{div}_{g}\left(|w|^{2} N\right)-|w|^{2} \operatorname{div}_{g}(N)\right] \mathrm{d} x \\
& =\frac{\lambda^{2}}{2} \int_{\Gamma}\left\langle|w|^{2} N, \mu\right\rangle_{g} \mathrm{~d} \Gamma-\frac{\lambda^{2}}{2} \int_{\Omega}|w|^{2} \operatorname{div}_{g}(N) \mathrm{d} x \\
& =\frac{\lambda^{2}}{2} \int_{\Gamma}|\tilde{u}|^{2} \mathrm{~d} \Gamma-\frac{\lambda^{2}}{2} \int_{\Omega}|w|^{2} \operatorname{div}_{g}(N) \mathrm{d} x
\end{aligned}
$$

By the Green's formula on the Riemannian manifold $(\bar{\Omega}, g)$ :

$$
\int_{\Omega} \Delta_{g} w N(\bar{w}) \mathrm{d} x=\int_{\Omega}\left\langle\nabla_{g} w, \nabla_{g} N(\bar{w})\right\rangle_{g} \mathrm{~d} x-\int_{\Gamma} N(\bar{w}) \frac{\partial w}{\partial \mu} \mathrm{d} \Gamma
$$

and Lemma 2.1 of [24], we have

$$
\begin{aligned}
\mathrm{RHS}= & -\int_{\Omega} \mathcal{A} w N(\bar{w}) \mathrm{d} x=\int_{\Omega} \Delta_{g} w N(\bar{w}) \mathrm{d} x-\int_{\Omega} \mathcal{T} w N(\bar{w}) \mathrm{d} x \\
= & -\int_{\Omega}\left\langle\nabla_{g} w, \nabla_{g} N(\bar{w})\right\rangle_{g} \mathrm{~d} x+\int_{\Gamma} N(\bar{w}) \frac{\partial w}{\partial \mu} \mathrm{d} \Gamma-\int_{\Omega} \mathcal{T} w N(\bar{w}) \mathrm{d} x \\
= & -\int_{\Omega}\left\langle\nabla_{g} w, \nabla_{g} N(\bar{w})\right\rangle_{g} \mathrm{~d} x+\int_{\Gamma}\left|\frac{\partial w}{\partial \mu}\right|^{2} \mathrm{~d} \Gamma-\int_{\Omega} \mathcal{T} w N(\bar{w}) \mathrm{d} x \\
= & -\int_{\Omega} D N\left(\nabla_{g} \bar{w}, \nabla_{g} w\right) \mathrm{d} x-\frac{1}{2} \int_{\Gamma}\left\langle\left|\nabla_{g} w\right|_{g}^{2} N, \mu\right\rangle_{g} \mathrm{~d} \Gamma+\frac{1}{2} \int_{\Omega}\left|\nabla_{g} w\right|_{g}^{2} \operatorname{div}_{g}(N) \mathrm{d} x \\
& +\int_{\Gamma}\left|\frac{\partial w}{\partial \mu}\right|^{2} \mathrm{~d} \Gamma-\int_{\Omega} \mathcal{T} w N(\bar{w}) \mathrm{d} x \\
= & -\int_{\Omega} D N\left(\nabla_{g} \bar{w}, \nabla_{g} w\right) \mathrm{d} x-\frac{1}{2} \int_{\Gamma}\left|\nabla_{g} w\right|_{g}^{2} \mathrm{~d} \Gamma+\int_{\Gamma}\left|\frac{\partial w}{\partial \mu}\right|^{2} \mathrm{~d} \Gamma \\
& +\frac{1}{2} \int_{\Omega}\left|\nabla_{g} w\right|_{g}^{2} \mathrm{div} g(N) \mathrm{d} x-\int_{\Omega} \mathcal{T} w N(\bar{w}) \mathrm{d} x \\
= & \frac{1}{2} \int_{\Gamma}\left(\left|\frac{\partial w}{\partial \mu}\right|^{2}-\left|\nabla_{T} \tilde{u}\right|_{g}^{2}\right) \mathrm{d} \Gamma+\int_{\Omega}\left[\left|\nabla_{g} w\right|_{g}^{2} \frac{\operatorname{div} g}{2}(N)\right. \\
& \left.-\int_{\Omega} \mathcal{T} w(\bar{w}) \mathrm{d} x, \quad D\left(\nabla_{g} \bar{w}, \nabla_{g} w\right)\right] \mathrm{d} x
\end{aligned}
$$

where we have used the fact $N(\bar{w})=\frac{\partial \bar{w}}{\partial \mu}$ on $\Gamma$, and $\nabla_{T}$ is the gradient of the tangential on $\Gamma:\left|\nabla_{g} w\right|_{g}^{2}=$ $\left|\frac{\partial w}{\partial \mu}\right|^{2}+\left|\nabla_{T} \tilde{u}\right|_{g}^{2}$ on $\Gamma$.

In what follows, we use the same $C$ to denote the constant that is independent of $\lambda$ and $w$ although it may have different values in different contexts. 
Letting LHS=RHS gives the identity:

$$
\int_{\Gamma}|F(\lambda) \tilde{u}|^{2} \mathrm{~d} \Gamma=\int_{\Gamma}|\tilde{u}|^{2} \mathrm{~d} \Gamma+\frac{1}{\lambda^{2}} \int_{\Gamma}\left|\nabla_{T} \tilde{u}\right|_{g}^{2} \mathrm{~d} \Gamma+\frac{1}{\lambda^{2}} f(\lambda)
$$

where

$$
f(\lambda)=\operatorname{Re}\left(\int_{\Omega} 2 D N\left(\nabla_{g} \bar{w}, \nabla_{g} w\right) \mathrm{d} x\right)-\int_{\Omega}\left(\lambda^{2}|w|^{2}+\left|\nabla_{g} w\right|_{g}^{2}\right) \operatorname{div}_{g}(N) \mathrm{d} x+\operatorname{Re}\left(2 \int_{\Omega} \mathcal{T} w N(\bar{w}) \mathrm{d} x\right)
$$

satisfying

$$
f(\lambda) \leq C\left(\lambda^{2}\|w\|_{L^{2}(\Omega)}^{2}+\left\|\left|\nabla_{g} w\right|_{g}\right\|_{L^{2}(\Omega)}^{2}\right) .
$$

Indeed,

$$
\begin{aligned}
f(\lambda) \leq & \int_{\Omega}\left|2 D N\left(\nabla_{g} \bar{w}, \nabla_{g} w\right)\right| \mathrm{d} x+\int_{\Omega}\left(\lambda^{2}|w|^{2}+\left|\nabla_{g} w\right|_{g}^{2}\right)\left|\operatorname{div}_{g}(N)\right| \mathrm{d} x+\int_{\Omega} 2|\mathcal{T} w| \cdot|N(\bar{w})| \mathrm{d} x \\
\leq & \int_{\Omega}\left|2\left\langle D_{\nabla_{g} \bar{w}} N, \nabla_{g} w\right\rangle_{g}\right| \mathrm{d} x+C\left(\lambda^{2}\|w\|_{L^{2}(\Omega)}^{2}+\left\|\left|\nabla_{g} w\right|_{g}\right\|_{L^{2}(\Omega)}^{2}\right)+\int_{\Omega}|\mathcal{T} w|^{2} \mathrm{~d} x+\int_{\Omega}|N(\bar{w})|^{2} \mathrm{~d} x \\
\leq & \int_{\Omega} 2\left|D \frac{\nabla_{g} \bar{w}}{\left|\nabla_{g} w\right|_{g}} N\right| \cdot\left|\nabla_{g} w\right|_{g}^{2} \mathrm{~d} x+C\left(\lambda^{2}\|w\|_{L^{2}(\Omega)}^{2}+\left\|\left|\nabla_{g} w\right|_{g}\right\|_{L^{2}(\Omega)}^{2}\right) \\
& +C\left\|\left|\nabla_{g} w\right|_{g}\right\|_{L^{2}(\Omega)}^{2}+\left\|\left|\nabla_{g} w\right|_{g}\right\|_{L^{2}(\Omega)}^{2} \\
\leq & C\left\|\left|\nabla_{g} w\right|_{g}\right\|_{L^{2}(\Omega)}^{2}+C\left(\lambda^{2}\|w\|_{L^{2}(\Omega)}^{2}+\left\|\left|\nabla_{g} w\right|_{g}\right\|_{L^{2}(\Omega)}^{2}\right)+C\left\|\left.|| \nabla_{g} w\right|_{g}\right\|_{L^{2}(\Omega)}^{2} \\
\leq & C\left(\lambda^{2}\|w\|_{L^{2}(\Omega)}^{2}+\left\|\left|\nabla_{g} w\right|_{g}\right\|_{L^{2}(\Omega)}^{2}\right)
\end{aligned}
$$

where we have used the facts $\sup _{\bar{\Omega}}\left|\operatorname{div}_{g}(N)\right| \leq C,|\mathcal{T} w| \leq C\left|\nabla_{g} w\right|_{g}$ and $|N(\bar{w})| \leq\left|\nabla_{g} w\right|_{g}$ when $|N|_{g} \leq 1$. $x \in \bar{\Omega}$

Next, multiply the right side of the first equation of (4.3) by $\bar{w}$ and integrate by parts to have

$$
\begin{aligned}
\int_{\Omega}-\mathcal{A} w \cdot \bar{w} \mathrm{~d} x & =\int_{\Omega} \Delta_{g} w \cdot \bar{w} \mathrm{~d} x-\int_{\Omega} \mathcal{T} w \cdot \bar{w} \mathrm{~d} x \\
& =-\int_{\Omega}\left\langle\nabla_{g} w, \nabla_{g} \bar{w}\right\rangle_{g} \mathrm{~d} x+\int_{\Gamma} \frac{\partial w}{\partial \mu} \overline{(\tilde{u})} \mathrm{d} \Gamma-\int_{\Omega} \mathcal{T} w \cdot \bar{w} \mathrm{~d} x
\end{aligned}
$$

This together with (4.3) gives

$$
\begin{aligned}
\operatorname{Re}\left(\langle F(\lambda) \tilde{u}, \tilde{u}\rangle_{L^{2}(\Gamma)}\right) & =\operatorname{Re}\left(\int_{\Gamma} \frac{1}{\lambda} \frac{\partial w}{\partial \mu} \overline{(\tilde{u})} \mathrm{d} \Gamma\right) \\
& =\frac{1}{\lambda} \int_{\Omega}\left\langle\nabla_{g} w, \nabla_{g} \bar{w}\right\rangle_{g} \mathrm{~d} x-\frac{1}{\lambda} \int_{\Omega} \mathcal{A} w \cdot \bar{w} \mathrm{~d} x+\frac{1}{\lambda} \operatorname{Re}\left(\int_{\Omega} \mathcal{T} w \cdot \bar{w} \mathrm{~d} x\right) \\
& =\frac{1}{\lambda} \int_{\Omega}\left|\nabla_{g} w\right|_{g}^{2} \mathrm{~d} x+\lambda \int_{\Omega}|w|^{2} \mathrm{~d} x+\frac{1}{\lambda} \operatorname{Re}\left(\int_{\Omega} \mathcal{T} w \cdot \bar{w} \mathrm{~d} x\right) .
\end{aligned}
$$


On the other hand, by the Cauchy-Schwartz inequality, one has

$$
\left|\operatorname{Re}\left(\int_{\Omega} \mathcal{T} w \cdot \bar{w} \mathrm{~d} x\right)\right| \leq C\left(\lambda \int_{\Omega}|w|^{2} \mathrm{~d} x+\frac{1}{\lambda} \int_{\Omega}\left|\nabla_{g} w\right|_{g}^{2} \mathrm{~d} x\right) .
$$

Hence

$$
\operatorname{Re}\left(\langle F(\lambda) \tilde{u}, \tilde{u}\rangle_{L^{2}(\Gamma)}\right) \geq\left(1-\frac{C}{\lambda}\right)\left(\lambda\|w\|_{L^{2}(\Omega)}^{2}+\frac{1}{\lambda}\left\|\left|\nabla_{g} w\right|_{g}\right\|_{L^{2}(\Omega)}^{2}\right) .
$$

Combining (4.11) and (4.12), we obtain

$$
\begin{aligned}
\frac{f(\lambda)}{\lambda^{2}} & \leq \frac{C}{\lambda}\left(\lambda\|w\|_{L^{2}(\Omega)}^{2}+\frac{1}{\lambda}\left\|\left.\nabla_{g} w\right|_{g}\right\|_{L^{2}(\Omega)}^{2}\right) \leq \frac{C}{\lambda-C} \operatorname{Re}\left(\langle F(\lambda) \tilde{u}, \tilde{u}\rangle_{L^{2}(\Gamma)}\right) \\
& \leq \frac{C}{2(\lambda-C)}\left(\|F(\lambda) \tilde{u}\|_{L^{2}(\Gamma)}^{2}+\|\tilde{u}\|_{L^{2}(\Gamma)}^{2}\right) .
\end{aligned}
$$

Finally, from inequality (4.13) and identity (4.10), we conclude that

$$
\lim _{\lambda \in \mathbb{R}, \lambda \rightarrow \infty}\|F(\lambda) \tilde{u}\|_{L^{2}(\Gamma)}^{2}=\|\tilde{u}\|_{L^{2}(\Gamma)}^{2} .
$$

Step 2. Putting

we deduce from (4.14) that

$$
G(\lambda) \tilde{u}:=\operatorname{Re}\left(\langle F(\lambda) \tilde{u}, \tilde{u}\rangle_{L^{2}(\Gamma)}\right), \quad \lambda>0,
$$

$$
\limsup _{\lambda \rightarrow \infty} G(\lambda) \tilde{u} \leq \limsup _{\lambda \rightarrow \infty}\|F(\lambda) \tilde{u}\|_{L^{2}(\Gamma)}\|\tilde{u}\|_{L^{2}(\Gamma)}=\|\tilde{u}\|_{L^{2}(\Gamma)}^{2} .
$$

Next, the formula (4.12) implies that

$$
\lim _{\lambda \rightarrow \infty}\|w\|_{L^{2}(\Omega)}^{2}=\lim _{\lambda \rightarrow \infty} \frac{\lambda\|w\|_{L^{2}(\Omega)}^{2}}{\lambda} \leq \lim _{\lambda \rightarrow \infty} \frac{\operatorname{Re}\left(\langle F(\lambda) \tilde{u}, \tilde{u}\rangle_{L^{2}(\Gamma)}\right)}{\lambda-C} \leq \lim _{\lambda \rightarrow \infty} \frac{\|\tilde{u}\|_{L^{2}(\Gamma)}^{2}}{\lambda-C}=0 .
$$

On the other hand, integrate the both sides of (4.9) over $\Omega$ to yield

$$
\begin{aligned}
\|\tilde{u}\|_{L^{2}(\Gamma)}^{2} & =\operatorname{Re}\left(\int_{\Omega} 2 w N(\bar{w}) \mathrm{d} x\right)+\int_{\Omega}|w|^{2} \operatorname{div}_{g}(N) \mathrm{d} x \\
& \leq \lambda\|w\|_{L^{2}(\Omega)}^{2}+\frac{1}{\lambda}\left\|\left|\nabla_{g} w\right|_{g}\right\|_{L^{2}(\Omega)}^{2}+C\|w\|_{L^{2}(\Omega)}^{2} \leq \frac{\lambda}{\lambda-C} G(\lambda) \tilde{u}+C\|w\|_{L^{2}(\Omega)}^{2}
\end{aligned}
$$

where we have used (4.12) and the fact $|N(\bar{w})| \leq\left|\nabla_{g} w\right|_{g}$ when $|N|_{g} \leq 1$. This together with the fact $\lim _{\lambda \rightarrow \infty}\|w\|_{L^{2}(\Omega)}^{2}=0$ gives

$$
\|\tilde{u}\|_{L^{2}(\Gamma)}^{2} \leq \liminf _{\lambda \rightarrow \infty} \frac{\lambda}{\lambda-C} G(\lambda) \tilde{u}=\liminf _{\lambda \rightarrow \infty} G(\lambda) \tilde{u}
$$

We have thus obtained that

$$
\lim _{\lambda \rightarrow \infty} G(\lambda) \tilde{u}=\|\tilde{u}\|_{L^{2}(\Gamma)}^{2}
$$

(4.8) then follows from (4.14) and (4.16). The proof is complete.

Acknowledgements. The authors would like to thank Professor P. F. Yao for his kind help in the proof of Theorem 1.2 and analogous referees for their helpful comments. 


\section{REFERENCES}

[1] K. Ammari, Dirichlet boundary stabilization of the wave equation. Asymptotic Anal. 30 (2002) 117-130.

[2] K. Ammari and M. Tucsnak, Stabilization of second order evolution equations by a class of unbounded feedbacks. ESAIM: COCV 6 (2001) 361-386.

[3] C.I. Byrnes, D.S. Gilliam, V.I. Shubov and G. Weiss, Regular linear systems governed by a boundary controlled heat equation. J. Dyn. Control Syst. 8 (2002) 341-370.

[4] A. Cheng and K. Morris, Well-posedness of boundary control systems. SIAM J. Control Optim. 42 (2003) 1244-1265.

[5] R.F. Curtain, The Salamon-Weiss class of well-posed infinite dimensional linear systems: a survey. IMA J. Math. Control Inform. 14 (1997) 207-223.

[6] R.F. Curtain and G. Weiss, Well-posedness of triples of operators (in the sense of linear systems theory), in Control and Estimation of Distributed Parameter Systems, F. Kappel, K. Kunisch and W. Schappacher Eds., Birkhäuser, Basel 91 (1989) $41-59$.

[7] R. Glowinski, J.W. He and J.L. Lions, On the controllability of wave models with variable coefficients: a numerical investigation. Comput. Appl. Math. 21 (2002) 191-225.

[8] B.Z. Guo and Y.H. Luo, Controllability and stability of a second order hyperbolic system with collocated sensor/actuator. Syst. Control Lett. 46 (2002) 45-65.

[9] B.Z. Guo and Z.C. Shao, Regularity of a Schrödinger equation with Dirichlet control and collocated observation. Syst. Control Lett. 54 (2005) 1135-1142.

[10] B.Z. Guo and Z.C. Shao, Regularity of an Euler-Bernoulli plate equation with Neumann control and collocated observation. J. Dyn. Control Syst. 12 (2006) 405-418.

[11] B.Z. Guo and X. Zhang, The regularity of the wave equation with partial Dirichlet control and collocated observation. SIAM J. Control Optim. 44 (2005) 1598-1613.

[12] L. Hörmander, The Analysis of Linear Partial Differential Operators III. Springer-Verlag, Berlin (1985).

[13] B. Kellogg, Properties of elliptic boundary value problems, in Mathematical Foundations of the Finite Elements Methods. Academic Press, New York (1972) Chapter 3.

[14] V. Komornik, Exact controllability and stabilization: The Multiplier Method. John Wiley and Sons. Ltd., Chichester (1994).

[15] I. Lasiecka and R. Triggiani, Uniform exponential energy decay of wave equations in a bounded region with $L^{2}\left(0, \infty ; L^{2}(\Gamma)\right)$ feedback control in the Dirichlet boundary conditions. J. Diff. Eqns. 66 (1987) 340-390.

[16] I. Lasiecka and R. Triggiani, The operator $B^{*} L$ for the wave equation with Dirichlet control. Abstract Appl. Anal. $N^{\circ} \mathbf{7}$ (2004) 625-634.

[17] I. Lasiecka, J.L. Lions and R. Triggiani, Nonhomogeneous boundary value problems for second order hyperbolic operators. $J$. Math. Pure Appl. 65 (1986) 149-192.

[18] R.B. Melrose and J. Sjöstrand, Singularities of boundary value problems I. Comm. Pure Appl. Math. 31 (1978) 593-617.

[19] R. Triggiani, Exact boundary controllability on $L^{2}(\Omega) \times H^{-1}(\Omega)$ of the wave equation with Dirichlet boundary control acting on a portion of the boundary $\partial \Omega$, and related problems. Appl. Math. Optim. 18 (1988) 241-277.

[20] M. Tucsnak and G. Weiss, How to get a conservative well-posed linear system out of thin air II, controllability and stability. SIAM J. Control Optim. 42 (2003) 907-935.

[21] G. Weiss, Transfer functions of regular linear systems I: characterizations of regularity. Trans. Amer. Math. Soc. 342 (1994) $827-854$.

[22] G. Weiss and R. Rebarber, Optimizability and estimatability for infinite-dimensional linear systems. SIAM J. Control Optim. 39 (2000) 1204-1232.

[23] G. Weiss, O.J. Staffans and M. Tucsnak, Well-posed linear systems-a survey with emphasis on conservative systems. Int. J. Appl. Math. Comput. Sci. 11 (2001) 7-33.

[24] P.F. Yao, On the observability inequalities for exact controllablility of wave equations with variable coefficients. SIAM J. Control Optim. 37 (1999) 1568-1599. 